\title{
Efficient Secure Two-Party Computation Using Symmetric Cut-and-Choose
}

\author{
Yan Huang ${ }^{1}$, Jonathan Katz ${ }^{1, \star}$, and David Evans ${ }^{2, \star \star}$ \\ 1 Dept. of Computer Science, University of Maryland \\ $\{j k a t z$, yhuang\}@cs.umd.edu \\ 2 Dept. of Computer Science, University of Virginia \\ evans@cs.virginia.edu
}

\begin{abstract}
Beginning with the work of Lindell and Pinkas, researchers have proposed several protocols for secure two-party computation based on the cut-and-choose paradigm. In current instantiations of this approach, one party generates $\kappa$ garbled circuits; some fraction of those are "checked" by the other party, and the remaining fraction are evaluated.

We introduce here the idea of symmetric cut-and-choose protocols, in which both parties generate $\kappa$ circuits to be checked by the other party. The main advantage of our technique is that $\kappa$ can be reduced by a factor of 3 while attaining the same statistical security level as in prior work. Since the number of garbled circuits dominates the costs of the protocol, especially as larger circuits are evaluated, our protocol is expected to run up to 3 times faster than existing schemes. Preliminary experiments validate this claim.
\end{abstract}

\section{Introduction}

Secure two-party computation was shown to be feasible in the late 1980s [358]. But it is only in the past 10 years that the research community has devoted significant efforts toward making such protocols practical. Work in this direction was spurred by the Fairplay paper 25, which implemented Yao's protocol for two-party computation with security in the semi-honest setting. More recent work 101211] has shown that Yao's protocol (in combination with other techniques) can be surprisingly efficient when semi-honest security is sufficient.

More desirable, of course, is to achieve security against malicious adversaries. While this is known to be feasible, in principle, using generic zero knowledge [8], a generic approach of this sort does not currently seem likely to result in efficient protocols even if specialized zero-knowledge proofs (as suggested in [15]) are used. The first technique to be explored for making efficient two-party computation protocols secure against malicious adversaries was the cut-and-choose paradigm. In that approach, roughly speaking, one party generates $\kappa$ garbled circuits (where $\kappa$ is a statistical security parameter); some fraction of those are "checked" by

\footnotetext{
* This work was supported by DARPA and NSF award \#1111599.

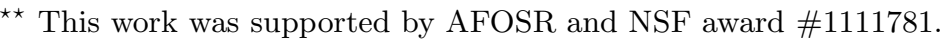

R. Canetti and J.A. Garay (Eds.): CRYPTO 2013, Part II, LNCS 8043, pp. 18-35, 2013.

(C) International Association for Cryptologic Research 2013
} 
the other party — who aborts if any misbehavior is detected - and the remaining fraction are evaluated with the results being used to derive the final output (we return to the exact mechanism for doing so in the next section). Cut-andchoose was used in a relatively naive way in 25] to give inverse-polynomial security. (In fact, the approach taken was later shown to be flawed [2616].) A rigorous analysis of the cut-and-choose paradigm was first given by Lindell and Pinkas 21, and their work was followed by numerous others exploring variations of this technique and their application to (ever more) efficient secure two-party computation [34|24|30|32|23/33|18].

In parallel with the above, other efficient approaches to achieving "full" malicious security in the two-party setting have also been explored. Approaches based on the IPS compiler [14] appear to have good asymptotic complexity [20], but seem challenging to implement (indeed, we are not aware of any implementations); other approaches 29,54 , have round complexity proportional to the depth of the circuit being evaluated. Another direction is to explore weaker security guarantees [12613], still against arbitrary malicious behavior. In the remainder of this paper we restrict our attention to protocols achieving the strongest notion of malicious security.

The critical question regarding the cut-and-choose approach is: how many garbled-circuit copies (namely, $\kappa$ ) are needed to ensure some desired security level? The value of $\kappa$ has the greatest impact on the efficiency of cut-andchoose protocols, especially as larger circuits $C$ are evaluated. The computational/communication complexity of such protocols is $O(\kappa \cdot k \cdot|C|)+\operatorname{poly}(n, k, s)$, where $k$ is a cryptographic security parameter and $n$ is the input length. Since $|C| \gg k, n$ (typical values are $k \approx 128$ and $n<1000$, while $|C| \approx 10^{9}$ in [18]), the importance of minimizing $\kappa$ is clear.

\subsection{Prior Work}

In previous applications of the cut-and-choose paradigm, one party (say, $P_{1}$ ) acts as the garbled-circuit generator and the other $\left(P_{2}\right)$ acts as the garbled-circuit evaluator; assume for simplicity that only $P_{2}$ gets output. If the oblivious-transfer (OT) protocol used is secure against malicious adversaries, the main issue is to ensure correctness of $P_{2}$ 's output. (Note, however, that correctness is closely connected with privacy, since $P_{1}$ can potentially carry out a selective failure attack in which the output of $P_{2}$ is correlated with $P_{2}$ 's input, in a way which would not be possible in an ideal evaluation of the function.) Toward that end, $P_{2}$ checks some number $c$ of the $\kappa$ circuits generated by $P_{1}$ to make sure they were constructed correctly. Assuming they were, the remaining $\kappa-c$ garbled circuits are evaluated by $P_{2}$, who then outputs the majority value of those circuits' results on each output wire. (This informal description omits various details, since we wish to focus on the cut-and-choose aspect of the protocols.)

From the above we see that a malicious $P_{1}$ can successfully cheat if they generate $b$ "bad" garbled circuits and (1) none of those bad garbled circuits is among the $c$ garbled circuits checked by $P_{1}$, and (2) of the remaining $\kappa-c$ garbled circuits being evaluated, half or more are bad. Doing the analysis, prior 
work [21 23] culminating in the work of Shelat and Shen [33] shows that using $\kappa$ garbled circuits yields security level $2^{-0.32 \kappa}$. Moreover, this bound was shown to be the best possible for a certain class of cut-and-choose approaches [33].

\subsection{Our Contribution}

We recast the cut-and-choose approach in a symmetric setting, where each party generates $\kappa$ garbled circuits to be checked by the other party. In doing so, we are motivated by work of Mohassel and Franklin [26] (see also [13]) who show how symmetric creation/evaluation of garbled circuits (but without any cut-andchoose) can be used to achieve security with only one bit of "disallowed" leakage against malicious adversaries. Here we show how to extend their approach to achieve the standard (i.e., "full") notion of malicious security.

After checking each other's garbled circuits, each party in our protocol evaluates the remaining garbled circuits of the other party, and then the results of both parties' evaluations are securely "combined" to yield the final output. Informally, a party outputs a value $v$ for some output wire of the circuit if and only if at least one of their own garbled circuits, and at least one of the garbled circuits generated by the other party, evaluate to $v$ on that wire. Since an honest party always generates correct garbled circuits, our analysis shows that correctness holds as long as at least one of the evaluated circuits provided by the other party is correct. (This is in contrast to one-sided cut-and-choose, where a majority of the evaluated circuits must be correct.) Thus, a malicious party can successfully cheat only if they generate exactly $\kappa-c$ "bad" garbled circuits, and none of those is checked by the other party. Setting $c=\kappa / 2$ (which minimizes the cheating probability), the probability of successful cheating is $\left(\begin{array}{c}\kappa \\ \kappa / 2\end{array}\right){ }^{-1}=2^{-\kappa+O(\log \kappa)}$. We can therefore achieve the same security level as previous work while reducing 1$]$ by roughly a factor of 3 .

As an added advantage, our protocol naturally supports having both parties receive output (an explicit concern of [33]), with no performance penalty if only one party should learn the output.

In concurrent work, Lindell [19] shows a different approach that achieves $2^{-\kappa}$ security using $\kappa$ circuits generated by only one of the parties.

\subsection{Outline of the Paper}

In Section 2 we review the cryptographic building blocks used in our protocol. We provide an overview of the protocol in Section 3 along with some intuition for why it is secure. In Section 4 we provide a formal description of our protocol, and we prove security in Section 5. In Appendix $\mathrm{A}$ we give some preliminary experimental results showing that we outperform the recent work of [18.

\footnotetext{
${ }^{1}$ To be clear: in our protocol each party generates $\kappa$ garbled circuits and so the total number of garbled circuits is $2 \kappa$. However, since this work is done in parallel by the two parties - in addition to whatever parallel processing is available on each user's own machine - and since the communication is symmetric, the "wall-clock time" of our protocol is expected to improve on previous protocols by up to a factor of 3 .
} 


\section{Notation and Building Blocks}

For simplicity, we describe our protocol using concrete (rather than asymptotic) notation. Nevertheless, it should be clear that our protocol can be cast in an asymptotic setting without difficulty.

Let $\mathbb{G}$ be a group of prime order $q$ with generator $g$. We assume the computational Diffie-Hellman $(\mathrm{CDH})$ problem is hard in $\mathbb{G}$. We let $H$ be a hash function that will be treated in the analysis as a random oracle. We let Com be a commitment scheme.

We use the standard definitions of secure two-party computation [7].

\subsection{Naor-Pinkas Oblivious Transfer}

In our protocol we do not use oblivious transfer as a "black box," but instead rely on specific details of the OT protocol used. Although several candidate OT protocols could be used, for concreteness and efficiency we use an OT protocol due to Naor and Pinkas 27] which we now describe.

Say we have a sender holding inputs $x_{0}, x_{1} \in\{0,1\}^{*}$, and a receiver holding input $b \in\{0,1\}$. In the first round, the sender chooses random $C \leftarrow \mathbb{G}$ and sends $C$ to the other party. The receiver picks $k \leftarrow \mathbb{Z}_{q}$, defines $h^{0}=g^{k}$ and $h^{1}=C / g^{k}$, and sends $h=h^{b}$ to the sender. In turn, the sender chooses $r \leftarrow \mathbb{Z}_{q}$ and sends $g^{r}, H\left(h^{r}\right) \oplus x_{0}, H\left((C / h)^{r}\right) \oplus x_{1}$ to the other party. The receiver recovers $x_{b}$ by computing $\left(g^{r}\right)^{k}$ and using the appropriate component of the sender's final message. Note that several independent OTs can share the same first message $C$.

This OT protocol is simulatable for a malicious receiver under the $\mathrm{CDH}$ assumption in the random oracle model. It achieves privacy (but is not known to be simulatable) against a malicious sender, and this suffices for our purposes. A variant of this protocol requires the receiver to give a (standard) perfect witnessindistinguishable proof of knowledge of $\log _{g} h$ or $\log _{g}(C / h)$ after sending $h$. We use this variant in our analysis since it simplifies the proof.

\subsection{Garbled Circuits}

We use a modification of standard garbled circuits [35]. Fix a function $f$ : $\{0,1\}^{n} \times\{0,1\}^{n} \rightarrow\{0,1\}^{n}$. We abstract the construction/evaluation of a garbled circuit for $f$ via algorithms GenGC, EvalGC with the following properties. GenGC is a randomized algorithm that takes as input $2 n$ input-wire labels $v_{1}^{0}, v_{1}^{1}, \ldots, v_{n}^{0}, v_{n}^{1} \in \mathbb{G}$ (corresponding to the first input of $f$ ), $2 n$ input-wire labels $v_{n+1}^{0}, v_{n+1}^{1}, \ldots, v_{2 n}^{0}, v_{2 n}^{1} \in\{0,1\}^{n}$ (corresponding to the second input of $f$ ), and $2 n$ output-wire labels $w_{1}^{0}, w_{1}^{1}, \ldots, w_{n}^{0}, w_{n}^{1} \in \mathbb{Z}_{q}$. It outputs a garbled circuit GC. Deterministic algorithm EvalGC takes as input GC and $2 n$ input-wire labels $v_{1}, \ldots, v_{2 n}$; it outputs $n$ values $b_{1}\left\|w_{1}, \ldots, b_{n}\right\| w_{n}$, with $b_{1}, \ldots, b_{n} \in\{0,1\}$. Note that EvalGC explicitly outputs wire labels in addition to bits.

Correctness requires that for any set of input/output-wire labels, any garbled circuit GC output by GenGC $\left(\left\{v_{i}^{0}, v_{i}^{1}\right\}_{i=1}^{2 n},\left\{w_{i}^{0}, w_{i}^{1}\right\}_{i=1}^{n}\right)$, and any $x, y \in\{0,1\}^{n}$ 
with $z=f(x, y)$, we have

$$
\operatorname{EvalGC}\left(\mathrm{GC},\left\{v_{i}^{x_{i}}\right\}_{i=1}^{n},\left\{v_{i}^{y_{i}}\right\}_{i=n+1}^{2 n}\right)=z_{1}\left\|w_{1}^{z_{1}}, \ldots, z_{n}\right\| w_{n}^{z_{n}} .
$$

Security requires a simulator SimGC such that for all $x, y$ with $z=f(x, y)$, any $v_{1}^{x_{1}}, \ldots, v_{n}^{x_{n}} \in \mathbb{G}, v_{n+1}^{y_{1}}, \ldots, v_{2 n}^{y_{n}} \in\{0,1\}^{n}$, and $w_{1}^{0}, w_{1}^{1}, \ldots, w_{n}^{0}, w_{n}^{1} \in \mathbb{Z}_{q}$, the distribution

$$
\left\{\begin{array}{c}
v_{1}^{1-x_{1}}, \ldots, v_{n}^{1-x_{n}} \leftarrow \mathbb{G} ; \\
v_{n+1}^{1-y_{1}}, \ldots, v_{2 n}^{1-y_{n}} \leftarrow\{0,1\}^{n} ; \\
\mathrm{GC} \leftarrow \operatorname{GenGC}\left(\left\{v_{i}^{0}, v_{i}^{1}\right\}_{i=1}^{2 n},\left\{w_{i}^{0}, w_{i}^{1}\right\}_{i=1}^{n}\right)
\end{array}:\left(\mathrm{GC},\left\{v_{i}^{x_{i}}\right\}_{i=1}^{n},\left\{v_{n+i}^{y_{i}}\right\}_{i=1}^{n}\right)\right\}
$$

is computationally indistinguishable from

$$
\left\{\mathrm{GC} \leftarrow \operatorname{SimGC}\left(x, z,\left\{v_{i}^{x_{i}}\right\}_{i=1}^{n},\left\{v_{n+i}^{y_{i}}\right\}_{i=1}^{n},\left\{w_{i}^{z_{i}}\right\}_{i=1}^{n}\right):\left(\mathrm{GC},\left\{v_{i}^{x_{i}}\right\}_{i=1}^{n},\left\{v_{n+i}^{y_{i}}\right\}_{i=1}^{n}\right)\right\} .
$$

In particular, this means (informally) that (1) given GC, $\left\{v_{i}^{x_{i}}\right\}_{i=1}^{n}$, and $\left\{v_{i}^{y_{n+i}}\right\}_{i=1}^{n}$, no information is leaked about $\left\{w_{i}^{1-z_{i}}\right\}_{i=1}^{n}$ where $z=f(x, y)$, and (2) this holds regardless of how the $\left\{v_{i}^{x_{i}}\right\}_{i=1}^{n},\left\{v_{n+i}^{y_{i}}\right\}_{i=1}^{n}$ are chosen (as long as the other inputwire labels are random). These properties are not standard, but are easily seen to hold by modifying the construction/proof from [22].

Note: We always let input wires $1, \ldots, n$ denote the inputs of the party generating the circuit. Thus, technically, $P_{1}$ generates garbled circuits for the function $f$, and $P_{2}$ generates garbled circuits for the function $f^{\prime}(y, x) \stackrel{\text { def }}{=} f(x, y)$.

\subsection{Verifiable Secret Sharing}

We use a notion of (non-interactive) verifiable secret sharing (VSS) that is weaker than the usual one in the literature. For our purposes, a $t$-out-of- $\kappa$ VSS scheme comprises three algorithms Share, Vrfy, Rec with the following functionality:

- Share takes input $s \in \mathbb{Z}_{q}$ and outputs $\kappa$ shares $w_{1}, \ldots, w_{\kappa} \in \mathbb{Z}_{q}$ and additional information pub.

- Vrfy takes as input the information pub, an index $i$, and a candidate share $w_{i} \in$ $\mathbb{Z}_{q}$. It outputs a bit, with 1 denoting validity.

- Rec takes as input pub and $t$ indices/shares $\left\{\left(i_{j}, w_{i_{j}}\right)\right\}_{j=1}^{t}$. It outputs a value $s \in \mathbb{Z}_{q}$.

We require that for any $s \in \mathbb{Z}_{q}$, any $w_{1}, \ldots, w_{\kappa}$, pub output by Share $(s)$, and any $i_{1}, \ldots, i_{t} \subset[\kappa]$, we have $\operatorname{Vrfy}\left(\operatorname{pub}, i, w_{i}\right)=1$ and $\operatorname{Rec}\left(\operatorname{pub},\left\{\left(i_{j}, w_{i_{j}}\right)\right\}_{j=1}^{t}\right)=s$.

We define a secrecy requirement for an honest dealer, and a verifiability requirement for honest receivers. Secrecy requires hardness of recovering a random secret $s$ given pub and at most $t-1$ shares. Formally, the following should be small for all efficient algorithms $\mathcal{A}$ and any $i_{1}, \ldots, i_{t-1}$ :

$$
\operatorname{Pr}\left[s \leftarrow \mathbb{Z}_{q} ;\left(\text { pub }, w_{1}, \ldots, w_{\kappa}\right) \leftarrow \operatorname{Share}(s): \mathcal{A}\left(\text { pub }, w_{i_{1}}, \ldots, w_{i_{t-1}}\right)=s\right]
$$


Verifiability requires that the dealer cannot generate pub and two different sets of valid shares that reconstruct to different secrets. Formally, the following is small for all efficient algorithms $\mathcal{A}$ :

$$
\begin{aligned}
& \operatorname{Pr}\left[\left(\text { pub },\left\{\left(i_{j}, w_{j}\right)\right\}_{j=1}^{t},\left\{\left(i_{j}^{\prime}, w_{j}^{\prime}\right)\right\}_{j=1}^{t}\right) \leftarrow \mathcal{A}\right. \\
& \forall j: \operatorname{Vrfy}\left(\operatorname{pub}, i_{j}, w_{j}\right)=1 \\
& \bigwedge \forall j: \operatorname{Vrfy}\left(\operatorname{pub}, i_{j}^{\prime}, w_{j}^{\prime}\right)=1 \\
& \left.\bigwedge \operatorname{Rec}\left(\operatorname{pub},\left\{\left(i_{j}, w_{j}\right)\right\}_{j=1}^{t}\right) \neq \operatorname{Rec}\left(\operatorname{pub},\left\{\left(i_{j}^{\prime}, w_{j}^{\prime}\right)\right\}_{j=1}^{t}\right)\right]
\end{aligned}
$$

Feldman VSS 6] satisfies the above properties under the discrete-logarithm assumption.

\section{High-Level Description of the Protocol}

At a high level, the protocol proceeds in the following stages:

1. Generate garbled circuits: Each party generates $\kappa$ garbled circuits along with their corresponding input-wire labels.

2. Oblivious transfer: Each party uses the Naor-Pinkas OT protocol (cf. Section 2.1) to obtain its input-wire labels for the garbled circuits constructed by the other party. This is done in such a way that a party must use the same effective input across all circuits.

3. "Cut-and-choose": Each party sends the garbled circuits they constructed to the other party. Using coin tossing, parties choose half of each of their circuits for checking. Then:

(a) For each of its check circuits, each party (1) sends all the input-wire labels for that circuit (to prove that the check circuit was constructed correctly) and (2) reveals all the values it used as the OT sender in step 2 (to prove that it used the correct input-wire labels in the OT execution corresponding to the check circuit).

(b) For each of its remaining circuits (the evaluation circuits), each party sends the input-wire labels corresponding to its own input.

4. Output determination: Each party evaluates the garbled circuits they received from the other party, using the input-wire labels obtained in steps 2 and $3(\mathrm{~b})$. For each output wire $i$ of the circuit, each party decides on output $z_{i} \in\{0,1\}$ iff at least one of the circuits they evaluated (that the other party constructed) gave output $z_{i}$ and at least one of the circuits the other party evaluated (that they constructed) gave output $z_{i}$.

We defer the details of step 4, and for now just assume it can be done. We also assume that if a party successfully passes the cut-and-choose step, then for at least one of that party's evaluation circuits (1) the evaluation circuit is constructed correctly and (2) the correct input-wire labels were used in the corresponding OT; this assumption holds except with probability at most $\left(\begin{array}{c}\kappa \\ \kappa / 2\end{array}\right)^{-1}$. 
The main issue to address is to ensure that a malicious party uses the same (effective) input in step 2 (when it obtains input-wire labels for its own input from the other party using OT) and for all the input-wire labels it sends in step 3(b) (for the garbled circuits that it generated). We achieve this by noting that when an honest receiver obtains the input-wire labels for its $i$ th input wire during the OT step, it sends a message $h_{i}$ for which (1) it knows $\log _{g} h_{i}$ when its effective input (on the $i$ th wire) is 0 , and (2) it $\operatorname{knows} \log _{g}\left(C / h_{i}\right)$ when its effective input (on the $i$ th wire) is 1 . We require the parties to use this same "template" for the input-wire labels corresponding to their own input in the garbled circuits they prepare. That is, for each garbled circuit and each input wire $i$ corresponding to an input of the circuit generator, the input-wire label $v_{i}^{0}$ corresponding to 0 is chosen such that $\log _{g} v_{i}^{0}$ is known, and the input-wire label $v_{i}^{1}$ corresponding to 1 is chosen such that $\log _{g}\left(C / v_{i}^{1}\right)$ is known. Moreover, this property is verified to hold (for the check circuits) during the cut-and-choose step. When sending its $i$ th input-wire label $v_{i}$ in step 3(b), each party must then also prove 2 that it knows $\log _{g}\left(v_{i} / h_{i}\right)$. This is reminiscent of a similar technique used by Shelat and Shen 33 to enforce input consistency among input-wire labels sent by the circuit generator; here, we extend it to enforce consistency also to the input-wire labels received as a circuit evaluator.

Given this - and still assuming step 4 can be carried out - one can informally verify that the protocol is secure. Assume for concreteness that $P_{2}$ is honest. Privacy of $P_{2}$ 's input is easy to see. As for correctness, $P_{2}$ constructed all its garbled circuits correctly and sent input-wire labels for its own input $y$ in all its evaluation circuits. In step $2, P_{1}$ obtained input-wire labels for $i t s$ own (effective) input $x$ in all of $P_{2}$ 's evaluation circuits. So all of $P_{2}$ 's garbled circuits that were evaluated by $P_{1}$ yield output $z \stackrel{\text { def }}{=} f(x, y)$. In the other direction, with high probability at least one of $P_{1}$ 's evaluation circuits $\mathrm{GC}^{*}$ was constructed correctly, and moreover the correct input-wire labels (for $P_{2}$ 's input) were used in the corresponding OT; thus, $P_{2}$ obtained the correct input-wire labels for its input $y$ in $\mathrm{GC}^{*}$. Furthermore, from the previous paragraph we know that the input-wire labels for $P_{1}$ 's input in $\mathrm{GC}^{*}$ correspond to the same input $x$ it used before. Thus, evaluation of $\mathrm{GC}^{*}$ by $P_{2}$ also yields $z=f(x, y)$, and thus $z$ will be the final output of $P_{2}$ in the protocol.

The missing piece is to show how to implement step 4 , and this is the most involved part of our protocol. The basic idea is for each party to choose a secret value $s_{i}^{b}$ for each output wire $i$ of the circuit and each possible value $b \in\{0,1\}$ that wire can take. Each secret is then split into $\kappa$ shares $w_{1, i}^{b}, \ldots, w_{\kappa, i}^{b}$ using a $(\kappa / 2+1)$-out-of- $\kappa$ secret-sharing scheme. Share $w_{j, i}^{b}$ is then used as the label corresponding to $b$ on the $i$ th output wire of the $j$ th garbled circuit. The net result is that for each output wire $i$ and bit $b$, the other party can reconstruct $s_{i}^{b}$ if and only if it learns $\kappa / 2+1$ of the shares corresponding to that wire and bit.

Note that $\kappa / 2$ shares of every wire and bit will be revealed as part of the cutand-choose phase. Assuming again that $P_{2}$ is honest, we thus have the following:

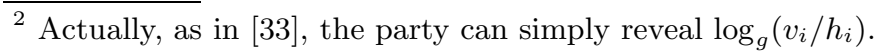


- As noted earlier, all of the garbled circuits that $P_{2}$ constructed will evaluate to the same value $z=f(x, y)$. This means that $P_{1}$ only learns shares corresponding to the secrets $s_{1}^{z_{1}}, \ldots, s_{n}^{z_{n}}$, and learns nothing about the remaining secrets $s_{1}^{1-z_{1}}, \ldots, s_{n}^{1-z_{n}}$. This gives $P_{2}$ a way to "test" whether the circuits it constructed (that were evaluated by $P_{1}$ ) resulted in output $z$ by checking which of each pair of secrets $P_{1}$ knows (e.g., using a secure equality test).

- In the opposite direction, as long as one of the garbled circuits constructed by $P_{1}$ (and evaluated by $P_{2}$ ) yields $z$, this will give $P_{2}$ one additional share of each of $\tilde{s}_{1}^{z_{1}}, \ldots, \tilde{s}_{n}^{z_{n}}$ (where we use $\tilde{s}$ here to denote that these secrets are chosen by $P_{1}$ ) and hence $P_{2}$ will be able to reconstruct each of those secrets. Note that it does not matter which garbled circuit evaluates to $z$, as any correctly constructed circuit that evaluates to $z$ reveals the requisite share.

One point omitted from the above discussion is that now it must be possible to check during the cut-and-choose phase that correct shares were used when constructing the garbled circuits. For this reason, we use verifiable secret sharing (see Section 2.3). We defer to the next section additional technical details of the protocol needed for the proof of security.

\section{Formal Specification of the Protocol}

Fix a function $f:\{0,1\}^{n} \times\{0,1\}^{n} \rightarrow\{0,1\}^{n}$ that parties $P_{1}$ and $P_{2}$ wish to compute over their respective inputs $x, y \in\{0,1\}^{n}$. We assume both parties learn the output, but it is easy to modify the protocol so that only one party learns the output. The protocol proceeds as follows.

1. $P_{1}$ chooses $C \leftarrow \mathbb{G}$ and sends it to $P_{2}$. Symmetrically, $P_{2}$ chooses $\widetilde{C} \leftarrow \mathbb{G}$ and sends it to $P_{1}$.

2. $P_{1}$ generates $4 n$ input-wire labels for each of $\kappa$ garbled circuits in the following way. For $j=1, \ldots, \kappa$, it chooses $a_{j, 1}^{0}, a_{j, 1}^{1}, \ldots, a_{j, n}^{0}, a_{j, n}^{1} \leftarrow \mathbb{Z}_{q}$ and sets the first $2 n$ input-wire labels of circuit $j$ to be of the form $\left\{v_{j, i}^{0}=g^{a_{j, i}^{0}}\right\}_{i=1}^{n}$ and $\left\{v_{j, i}^{1}=\widetilde{C} / g^{a_{j, i}^{1}}\right\}_{i=1}^{n}$. It chooses the next $2 n$ input-wire labels of circuit $j$ uniformly as $v_{i n+1}^{0}, v_{j, n+1}^{1}, \ldots, v_{j, 2 n}^{0}, v_{j, 2 n}^{1} \leftarrow\{0,1\}^{n}$.

Symmetrically $P_{2}$ generates $4 n$ input-wire labels $\widetilde{v}_{j, 1}^{0}, \widetilde{v}_{j, 1}^{1}, \ldots, \widetilde{v}_{j, 2 n}^{0}, \widetilde{v}_{j, 2 n}^{1}$ for $j=1, \ldots, \kappa$.

Each party then uses Naor-Pinkas OT to obtain the input-wire labels corresponding to its own input in the circuits generated by the other party. I.e., for $i=1, \ldots, n$ party $P_{1}$ chooses $k_{i} \leftarrow \mathbb{Z}_{q}$, generates $\left(h_{i}^{0}, h_{i}^{1}\right)=\left(g^{k_{i}}, \widetilde{C} / g^{k_{i}}\right)$, and sends $h_{i} \stackrel{\text { def }}{=} h_{i}^{x_{i}}$ to $P_{2}$. Then $P_{2}$ generates $\kappa$ independent responses as in the Naor-Pinkas protocol, using inputs $\left(\widetilde{v}_{j, n+i}^{0}, \widetilde{v}_{j, n+i}^{1}\right)$ in the $j$ th such instance where, recall, $\widetilde{v}_{j, n+i}^{b}$ denotes the label corresponding to bit $b$ on the $(n+i)$ th input wire in the $j$ th garbled circuit. $P_{1}$ recovers $\widetilde{v}_{1, n+i}^{x_{i}}, \ldots, \widetilde{v}_{\kappa, n+i}^{x_{i}}$. $P_{2}$ acts symmetrically to obtain $v_{1, n+i}^{y_{i}}, \ldots, v_{\kappa, n+i}^{y_{i}}$ for $i=1, \ldots, n$.

${ }^{3}$ Recall that the first $n$ input wires always denote the inputs of the party generating the circuit. 
3. For $i \in\{1, \ldots, n\}$ and $b \in\{0,1\}$, party $P_{1}$ chooses $s_{i}^{b} \leftarrow \mathbb{Z}_{q}$ and generates a $(\kappa / 2+1)$-out-of- $\kappa$ secret sharing $\left(\operatorname{pub}_{i}^{b}, w_{1, i}^{b}, \ldots, w_{\kappa, i}^{b}\right) \leftarrow \operatorname{Share}\left(s_{i}^{b}\right)$. It uses $w_{j, i}^{b}$ as the label for bit $b$ on the $i$ th output wire in the $j$ th circuit, i.e., for $j=1, \ldots, \kappa$ it computes the garbled circuit

$$
\mathrm{GC}_{j}=\operatorname{GenGC}\left(\left\{v_{j, i}^{0}, v_{j, i}^{1}\right\}_{i=1}^{2 n},\left\{w_{j, i}^{0}, w_{j, i}^{1}\right\}_{i=1}^{n}\right) .
$$

It sends $\left\{\mathrm{GC}_{j}\right\}_{j=1}^{\kappa}$ and $\left\{\operatorname{pub}_{i}^{0}, \operatorname{pub}_{i}^{1}\right\}_{i=1}^{n}$ to $P_{2}$.

$P_{2}$ acts symmetrically to obtain $\widetilde{s}_{i}^{b}$ and $\left(\widetilde{\operatorname{pub}}_{i}^{b}, \widetilde{w}_{1, i}^{b}, \ldots, \widetilde{w}_{\kappa, i}^{b}\right)$ and to generate $\widetilde{\mathrm{GC}}_{j}$; it sends $\left\{\widetilde{\mathrm{GC}}_{j}\right\}_{j=1}^{\kappa}$ and $\left\{\widetilde{\operatorname{pub}}_{i}, \widetilde{\operatorname{pub}}_{i}^{1}\right\}_{i=1}^{n}$ to $P_{1}$.

4. For $j=1, \ldots, \kappa$ and $i=1, \ldots, n$, party $P_{1}$ commits to the input-wire labels $v_{j, i}^{0}$ and $v_{j, i}^{1}$ corresponding to its own input, in random permuted order. Let ComSet $_{j, i}$ denote the resulting pair of commitments. $P_{2}$ acts symmetrically.

5. The parties run secure coin-tossing protocols to generate strings $\mathcal{J}, \widetilde{\mathcal{J}} \in$ $\{0,1\}^{\kappa}$ that are each uniform among strings containing exactly $\kappa / 2$ ones 4 These are interpreted in the natural way as subsets of $\{1, \ldots, \kappa\}$ of size $\kappa / 2$. $\widetilde{J}$ is used to check the garbled circuits constructed by $P_{1}$. Specifically, for $j=1, \ldots, \kappa$ :

(a) If $j \in \widetilde{J}$ the $j$ th circuit is a check circuit. Then $P_{1}$ sends $\left\{a_{j, i}^{0}, a_{j, i}^{1}\right\}_{i=1}^{n}$, $\left\{v_{j, i}^{0}, v_{j, i}^{1}\right\}_{i=n+1}^{2 n},\left\{w_{j, i}^{0}, w_{j, i}^{1}\right\}_{i=1}^{n}$, and the randomness it used to generate $\mathrm{GC}_{j}$. It also reveals the sender-randomness it used in all the OTs corresponding to the $j$ th circuit, and opens both commitments in ComSet $_{j, i}$ for $i=1, \ldots, n$.

$P_{2}$ sets $v_{j, i}^{0}=g^{a_{j, i}^{0}}$ and $v_{j, i}^{1}=\widetilde{C} / g^{a_{j, i}^{1}}$ for $i=1, \ldots, n$. It re-generates the $j$ th garbled circuit and verifies that it matches $\mathrm{GC}_{j}$. It verifies that $\left\{v_{j, i}^{0}, v_{j, i}^{1}\right\}_{i=n+1}^{2 n}$ were used in the OTs for the $j$ th circuit, and that the commitments in $\operatorname{ComSet}_{j, i}$ open to $\left\{v_{j, i}^{0}, v_{j, i}^{1}\right\}$ in some order. Finally, it checks that $\operatorname{Vrfy}\left(\operatorname{pub}_{i}^{b}, j, w_{j, i}^{b}\right)=1$ for $i=1, \ldots, n$ and $b \in\{0,1\}$. It aborts if any of these fail.

(b) If $j \notin \widetilde{J}$ the $j$ th circuit is an evaluation circuit. In this case, $P_{1}$ sends $\left(v_{j, 1}, \ldots, v_{j, n}\right) \stackrel{\text { def }}{=}\left(v_{j, 1}^{x_{1}}, \ldots, v_{j, n}^{x_{n}}\right)$ (i.e., the wire labels corresponding to $P_{1}$ 's input in the $j$ th circuit) to $P_{2}$. It also opens the commitment in ComSet $_{j, i}$ that corresponds to $v_{j, i}$. Finally, it sends $\log _{g}\left(v_{j, 1} / h_{1}\right), \ldots$, $\log _{g}\left(v_{j, n} / h_{n}\right)$. (Recall that $h_{1}, \ldots, h_{n}$ are the values used by $P_{1}$ when acting as receiver in the Naor-Pinkas OT protocol.)

$P_{2}$ checks that one of the commitments in ComSet ${ }_{j, i}$ opens to $v_{j, i}$, and verifies the discrete logarithms sent by $P_{1}$. It aborts if any inconsistencies are found.

Symmetrically, the parties use $\mathcal{J}$ to check the garbled circuits constructed by $P_{2}$.

${ }^{4}$ This can be implemented easily by using a standard coin-tossing protocol to generate polynomially many uniform bits, and then using those bits as the random coins for applying a Knuth shuffle to the string $0^{\kappa / 2} 1^{\kappa / 2}$. 
6. For each evaluation circuit $j$ of $P_{2}$, party $P_{1}$ evaluates $\widetilde{\mathrm{GC}}_{j}$ using the inputwire labels it obtained in steps 2 and 5. By doing so, it learns $n$ values $\widetilde{b}_{j, 1}\left\|\widetilde{w}_{j, 1}, \ldots, \widetilde{b}_{j, n}\right\| \widetilde{w}_{j, n}$.

For $i=1, \ldots, n$ and $b \in\{0,1\}$, party $P_{1}$ tries to recover $5 \widetilde{s}_{i}^{b}$. To do so, it finds an evaluation circuit $j$ for which $\widetilde{b}_{j, i}=b$ and $\widetilde{w}_{j, i}$ is a valid share of $\widetilde{s}_{i}^{b}$ (i.e., $\left.\operatorname{Vrfy}\left(\widetilde{\operatorname{pub}}_{i} \widetilde{b}_{j, i}, j, \widetilde{w}_{j, i}\right)=1\right)$. If no such $j$ exists, it chooses $t_{i}^{b} \leftarrow \mathbb{Z}_{q}$. Otherwise, it computes $t_{i}^{b}$ by running Rec using $\widetilde{\operatorname{pub}_{i}} \widetilde{b}_{j, i}$, the $\kappa / 2$ shares $\left\{\left(k, \widetilde{w}_{k, i}^{\widetilde{b}_{j, i}}\right)\right\}_{k \in \mathcal{J}}$ it learned in step [5] and the additional share $\left(j, \widetilde{w}_{j, i}\right)$.

$P_{2}$ acts symmetrically to compute $\widetilde{t}_{i}^{0}, \widetilde{t}_{i}^{1}$ for $i=1, \ldots, n$.

7. For $i=1, \ldots, n$, the parties do the following: Run a secure equality test, with $P_{1}$ using input $s_{i}^{0} \| t_{i}^{0}$ and $P_{2}$ using input $\widetilde{t}_{i}^{0} \| \widetilde{s}_{i}^{0}$. If the result is 1 , each party sets $z_{i}=0$ and goes to the next $i$. Otherwise, the parties run a second equality test with $P_{1}$ using input $s_{i}^{1} \| t_{i}^{1}$ and $P_{2}$ using input $\widetilde{t}_{i}^{1} \| \widetilde{s}_{i}^{1}$. If the result is 1 , each party sets $z_{i}=1$ and goes to the next $i$. If neither equality test succeeds for some $i$ then cheating is detected and the parties abort.

Assuming no abort has occurred, each party then outputs $z=z_{1} \cdots z_{n}$.

\subsection{Optimizations}

For simplicity in our proof of security in the following section, we analyze the protocol as presented above. However, we observe that the following optimizations can be applied to the protocol (and the reader can verify that the proof can be suitably modified for each of these).

Naor-Pinkas OT. We assume a variant of Naor-Pinkas OT is used in which the receiver gives a witness-indistinguishable (WI) proof of knowledge that its message was computed correctly (see Section 2.1). This is used in our proof to extract the receiver's input. In fact, as shown in [27, such WI proofs are not necessary and extraction can be done using the random-oracle queries of the receiver. The same is true in our setting, though it complicates the presentation of the proof.

Secure Coin Tossing. In the (programmable) random-oracle model, very efficient coin tossing is possible since it is trivial to construct an equivocal and extractable commitment scheme.

Secure Equality Testing. In our proof, we assume a hybrid world in which the parties have access to an ideal functionality for equality testing; equivalently (relying on standard composition theorems [3]), we assume that the equality test is done using a fully secure protocols for this task.

In fact, using a fully secure equality test is overkill for our purposes. Instead, we can use a different approach that is very efficient in the random-oracle model. First, assume the VSS scheme has the stronger property of indistinguishability, i.e., given pub and $t-1$ shares of a uniform secret $s \in\{0,1\}^{n}$, it is hard to

\footnotetext{
${ }^{5}$ In an honest execution, only one of $\widetilde{s}_{i}^{0}$ or $\widetilde{s}_{i}^{1}$ will be recovered.
} 
distinguish $s$ from an independent uniform value $s^{\prime} \in\{0,1\}^{n}$. (Any VSS scheme satisfying the unpredictability requirement from Section 2.3 can be modified to achieve this stronger notion in the random-oracle model by simply hashing the secret.) Then, rather than performing an equality test using values $s_{i}^{0} \| t_{i}^{0}$ and $\widetilde{t}_{i}^{0} \| \widetilde{s}_{i}^{0}$ (resp., $s_{i}^{1} \| t_{i}^{1}$ and $\widetilde{t}_{i}^{1} \| \widetilde{s}_{i}^{1}$ ) as before, the parties now carry out an equality test on values $s_{i}^{0} \oplus t_{i}^{0}$ and $\widetilde{t}_{i}^{0} \oplus \widetilde{s}_{i}^{0}$ (resp., $s_{i}^{1} \oplus t_{i}^{1}$ and $\widetilde{t}_{i}^{1} \oplus \widetilde{s}_{i}^{1}$ ). At this point, we observe that a full-fledged equality test is not needed since (1) the honest party's input to the equality test is either known to the malicious party or is (indistinguishable from) uniform, and (2) in either case, it is ok if the honest party's input to the equality test is leaked to the other party after equality is checked. Thus, it suffices to use a "cheap" equality test in which $P_{1}$ (resp., $P_{2}$ ) commits to, e.g., $s_{i}^{0} \oplus t_{i}^{0}$ (resp., to $\widetilde{t}_{i}^{0} \oplus \widetilde{s}_{i}^{0}$ ) using an extractable and equivocal commitment scheme (which is easily constructed in the random-oracle model), and then each party decommits and checks equality of the decommitted results in the clear.

Saving Bandwidth. Following an observation in [9], we can modify the way we do cut-and-choose as follows: Parties construct their $j$ th garbled circuit by choosing a random seed seed ${ }_{j}$ and using that seed to generate certain (pseudo)random choices they need for constructing that circuit. (In our case, this would mean us-

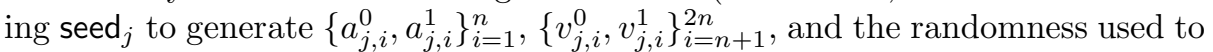
generate $\mathrm{GC}_{j}$.) Then, in step 3, the parties send the $h a s h \mathrm{hGC}_{j}=H\left(\mathrm{GC}_{j}\right)$ in place of $\mathrm{GC}_{j}$. If $j$ is a check circuit then seed $j$ is sent; the other party re-generates $\mathrm{GC}_{j}$ and verifies that $H\left(\mathrm{GC}_{j}\right)=\mathrm{hGC}_{j}$. If $j$ is an evaluation circuit then $\mathrm{GC}_{j}$ is sent and the other party checks that $H\left(\mathrm{GC}_{j}\right)=\mathrm{hGC}_{j}$. Since $\left|\operatorname{seed}_{j}\right|+\left|\mathrm{hGC}_{j}\right| \ll\left|\mathrm{GC}_{j}\right|$, this has the effect of reducing the bandwidth in steps 3 and 5 (which dominate the bandwidth of the entire protocol) by roughly half.

Batch Verification. We can use batch verification [2 when simultaneously verifying validity of shares in step 5(a) (assuming Feldman VSS is used) and the discrete logarithms in step 5 (b).

Efficient Garbled Circuits. Our protocol is fully compatible with existing optimizations for garbled circuits such as garbled-row reduction [28] and the free-XOR technique [17] 6

\section{Proof of Security}

Theorem 1. Under the assumptions outlined in Section 2, and modeling $H$ as a random oracle, the protocol in the previous section securely computes $f$ in the presence of malicious adversaries.

${ }^{6}$ We cannot apply the free-XOR optimization at first-level gates because of the way the circuit generator chooses the input-wire labels. However, the free-XOR method can be used at all lower levels of the circuit. 
Since we are not in an asymptotic setting, technically speaking "secure" is not well-defined. In the proof below, all steps introduce a computational security factor (which can be set as small as desired by setting the cryptographic security parameter large enough) except for one step which introduces a statistical security factor of $\left(\begin{array}{c}\kappa \\ \kappa / 2\end{array}\right)^{-1}=2^{-\kappa+O(\log \kappa)}$.

All our assumptions are standard, and can be based on the CDH assumption in $\mathbb{G}$. We remark that the only place the random oracle is used is for the NaorPinkas OT. It would be possible to remove the random oracle by switching, e.g., to the OT protocol of [31] (and modifying the rest of the protocol accordingly). Although this would impact the efficiency, the effect would be proportional to the input length and not the size of the circuit being computed.

Proof. We analyze the protocol in a hybrid world in which the parties have access to ideal functionalities for coin tossing and equality testing. Using standard composition theorems [3], this implies security when those sub-routines are instantiated using secure protocols for those tasks.

Since the protocol is symmetric, we assume without loss of generality that $P_{1}$ is malicious. Let $y$ denote the input of $P_{2}$. We define a sequence of experiments, beginning with the real execution of the protocol between $P_{1}$ and $P_{2}$ (in the hybrid world discussed above) and ending with an ideal execution involving a simulator $\mathcal{S}$ playing the role of the first party and interacting with a trusted party computing $f$. We show that each experiment is indistinguishable from the one before it, taking into account both the view/output of the malicious party and the output of $P_{2}$.

Experiment 0. This is the real execution of the protocol (in the hybrid world discussed above) between $P_{1}$ and the honest $P_{2}$ holding input $y$.

Experiment 1. Here we change the way $P_{2}$ behaves when acting as OT sender in step 2 and when sending commitments in step 4. First of all, we now pick $\mathcal{J}$ at the outset of the experiment. This defines the check circuits and evaluation circuits for $P_{2}$. Next, in each instance $i$ in which $P_{1}$ acts as OT receiver in step 2 and sends message $h_{i}$, we extract (using the WI proof of knowledge) either $\log _{g} h_{i}$ or $\log _{g}\left(\widetilde{C} / h_{i}\right)$. In the former case we set $x_{i}=0$ and in the latter case we set $x_{i}=1$. Then, when computing the $\kappa$ responses for the $i$ th OT, in each response that corresponds to an evaluation circuit $j$ of $P_{2}$ we continue to use $\widetilde{v}_{j, n+i}^{x_{i}}$ but we replace $\widetilde{v}_{j, n+i}^{1-x_{i}}$ with the all-0 string. (Responses that correspond to check circuits of $P_{2}$ are treated exactly as before.)

In addition, for each evaluation circuit $j$ of $P_{2}$ and $i=1, \ldots, n$, we now set $\operatorname{ComSet}_{j, i}=\left\{\operatorname{Com}\left(\widetilde{v}_{j, i}^{y_{i}}\right), \operatorname{Com}(g)\right\}$, in random permuted order.

Indistinguishability of Experiments 0 and 1 follows easily from the security of Naor-Pinkas OT (based on the CDH assumption in the random-oracle model) and computational hiding of Com.

Experiment 2. Now we generate all the evaluation circuits of $P_{2}$ using the garbled-circuit simulator SimGC. In more detail: after extracting $P_{1}$ 's effective input $x$ as in the previous experiment, we compute $z=f(x, y)$. In step 3 , once 
the $\left\{\widetilde{w}_{j, i}^{b}\right\}$ have been determined we compute for every evaluation circuit $j$ the simulated garbled circuit $7 \widetilde{\mathrm{GC}}_{j} \leftarrow \operatorname{SimGC}\left(x, z,\left\{\widetilde{v}_{j, i}^{y_{i}}\right\}_{i=1}^{n},\left\{\widetilde{v}_{j, n+i}^{x_{i}}\right\}_{i=1}^{n},\left\{\widetilde{w}_{j, i}^{z_{i}}\right\}_{i=1}^{n}\right)$. The remainder of the experiment is exactly as in Experiment 1 .

Indistinguishability of Experiments 1 and 2 follows from security of the garbled-circuit simulation algorithm as defined in Section 2.2 .

Note that in Experiment 2, we no longer use $\left\{\widetilde{v}_{j, i}^{1-y_{i}}\right\}_{i=1}^{n},\left\{\widetilde{v}_{j, n+i}^{1-x_{i}}\right\}_{i=1}^{n}$, or $\left\{\widetilde{w}_{j, i}^{1-z_{i}}\right\}_{i=1}^{n}$ for any evaluation circuit $j$ of $P_{2}$.

Experiment 3. This is the same as the previous experiment, except that now when performing the $i$ th pair of equality tests we proceed as follows: if $z_{i}=1$, we return 0 to both parties in the first equality test; if $z_{i}=0$, we return 0 to both parties in the second equality test (if run).

Indistinguishability of this experiment from Experiment 2 follows from secrecy of VSS. Specifically, for $i=1, \ldots, n$ only $\widetilde{\operatorname{pub}}_{i}^{1-z_{i}}$ and $\kappa / 2$ shares of the secret $\widetilde{s}_{i}^{1-z_{i}}$ are used throughout the entire experiment before the equality tests. Thus, the probability (in Experiment 2) that $P_{1}$ can make any of the equality tests corresponding to $1-z_{i}$ return 1 is negligible.

Experiment 4. If $P_{1}$ successfully responds to the "challenge" $\widetilde{\mathcal{J}}$ chosen during the cut-and-choose step, we repeatedly rewind $P_{1}$ in an attempt to find a $\tilde{\mathcal{J}}^{\prime} \neq$ $\widetilde{\mathcal{J}}$ for which $P_{1}$ also responds correctly 8 If no such $\widetilde{\mathcal{J}}^{\prime}$ is found, output fail. Otherwise, re-send the original challenge $\widetilde{\mathcal{J}}$ and continue as in the previous experiment.

The only difference between this experiment and the previous one occurs in case $P_{1}$ responds correctly to only a single challenge $\widetilde{\mathcal{J}}$ and that challenge happens to be the one chosen during the experiment. This can occur with probability at most $1 /\left(\begin{array}{c}\kappa \\ \kappa / 2\end{array}\right)$.

Experiment 5. We now change how we compute $\tilde{t}_{i}^{z_{i}}$ for all $i$. (Recall that $\tilde{t}_{i}^{z_{i}}$ represents $P_{2}$ 's guess for $P_{1}$ 's secret $s_{i}^{z_{i}}$.) Assuming $P_{1}$ answers two different challenges $\tilde{\mathcal{J}}, \tilde{\mathcal{J}}^{\prime}$ correctly, there is some $j^{*} \in\{1, \ldots, \kappa\}$ such that $j^{*}$ is an evaluation circuit with respect to $\widetilde{\mathcal{J}}$ but a check circuit with respect to $\tilde{\mathcal{J}}^{\prime}$. For any such $j^{*}$, we reconstruct $s_{i}^{z_{i}}$ using the share $w_{j^{*}, i}^{z_{i}}$ sent by $P_{1}$ when answering challenge $\tilde{\mathcal{J}}^{\prime}$, along with the $\kappa / 2$ other shares of $s_{i}^{z_{i}}$ that were sent by $P_{1}$ when answering challenge $\widetilde{\mathcal{J}}$. We then set $\tilde{t}_{i}^{z_{i}}=s_{i}^{z_{i}}$ and use that value in the relevant equality test later.

We claim that this experiment is indistinguishable from the previous one; this is the crux of the proof. To prove this, we show that the shares $\left\{w_{j^{*}, i}^{z_{i}}\right\}_{i=1}^{n}$ computed in Experiment 5 are, except with negligible probability, the same

\footnotetext{
${ }^{7}$ Recall that the first $n$ input wires always denote the inputs of the party generating the circuit, so in this case correspond to input $y$.

${ }^{8}$ We use standard techniques in order to ensure that the experiment runs in expected polynomial time. Specifically, in parallel with rewinding $P_{1}$ and sending a random challenge $\widetilde{\mathcal{J}}^{\prime} \neq \widetilde{\mathcal{J}}$ we also enumerate over all possible $\widetilde{\mathcal{J}}^{\prime}$; we output fail if we find that $\widetilde{\mathcal{J}}$ is the only challenge to which $P_{1}$ responds correctly.
} 
shares that $P_{2}$ obtains by evaluating circuit $\mathrm{GC}_{j *}$ in Experiment 4 . Verifiability of the secret-sharing scheme then implies that, except with negligible probability, the same values $\left\{\widetilde{t}_{i}^{z_{i}}\right\}_{i=1}^{n}$ are computed in both experiments (namely, even if in Experiment 4 a valid share from an evaluation circuit other than $j^{*}$ is used by $P_{2}$ to reconstruct some $s_{i}^{z_{i}}$ ).

Fix $i$. To see that the same share $w_{j^{*}, i}^{z_{i}}$ is computed in each experiment, observe that in Experiment 4 the share $w_{j^{*}, i}^{z_{i}}$ is computed by evaluating garbled circuit $\mathrm{GC}_{j^{*}}$ using input-wire labels for $P_{2}$ 's input that $P_{2}$ obtains from the OTs corresponding to circuit $j^{*}$, and input-wire labels for $P_{1}$ 's input that were sent by $P_{1}$ in step [5. Because $P_{1}$ responds correctly to challenge $\widetilde{\mathcal{J}}^{\prime}$, in which $j^{*}$ is a check circuit, we know that: (1) $\mathrm{GC}_{j^{*}}$ is correctly constructed; (2) the input-wire labels that $P_{2}$ obtained from the OTs are correct labels for $\mathrm{GC}_{j *}$ that correspond to $P_{2}$ 's input $y$; $(3)$ the input-wire labels for its own input that $P_{1}$ sends must be correct labels for $\mathrm{GC}_{j^{*}}$ (this follows from binding of the commitments in $\left\{\operatorname{ComSet}_{j^{*}, i}\right\}_{i=1}^{n}$ ) and moreover must correspond to the same effective input $x$ defined by $P_{1}$ 's execution as OT receiver (otherwise we obtain a discrete logarithm of the random group element $\widetilde{C}$ ). Since $\mathrm{GC}_{j^{*}}$, when evaluated on input-wire labels corresponding to $x$ and $y$, yields the share $w_{j^{*}, i}^{z_{i}}$ on the $i$ th output wire, we are done.

In Experiment 5 none of $P_{1}$ 's evaluation circuits need to be evaluated by $P_{2}$. Moreover, $P_{2}$ no longer needs to compute its output in any of the OTs in which it acts as receiver.

Experiment 6. In the previous experiment, when $P_{2}$ acts as OT receiver it sends $\widetilde{h}_{i}$ with either $\log _{g} \widetilde{h}_{i}$ or $\log _{g}\left(C / \widetilde{h}_{i}\right)$ known (depending on $\left.y_{i}\right)$. The inputwire labels $\left\{\widetilde{v}_{j, i}^{y_{i}}\right\}_{i=1}^{n}$ (when $j$ is an evaluation circuit) are chosen in a similar way. In this experiment, for $i=1, \ldots, n$ we choose $\widetilde{h}_{i}$ uniform with $\log _{g} \widetilde{h}_{i}$ known so that we are simply running the OT execution honestly using input 0. Similarly, choose $\widetilde{v}_{j, i}^{y_{i}}$ uniform with $\log _{g} \widetilde{v}_{j, i}^{y_{i}}$ known for every evaluation circuit $j$. (Note that this allows $P_{2}$ to reveal $\log _{g}\left(\widetilde{v}_{j, i} / \widetilde{h}_{i}\right)$ in step 5 for every evaluation circuit $j$.)

This experiment is distributed identically to the previous experiment, since $g^{k}$ and $C / g^{k}$ (where $k$ is uniform in each case) have the same distribution. $\left(P_{2}\right.$ also gives a WI proof of knowledge of either $\log _{g} \widetilde{h}_{i}$ or $\log _{g}\left(C / \widetilde{h}_{i}\right)$, but we assume a perfect WI proof is used.)

To conclude, we observe that Experiment 6 can equivalently be described in terms of an ideal-world execution in which the honest $P_{2}$ and a simulator $\mathcal{S}$ (playing the role of the first party, and running $P_{1}$ as a subroutine) interact with a trusted party computing $f$. Namely, $\mathcal{S}$ works as follows:

1. Choose $\mathcal{J}$ in advance; this defines the check circuits and the evaluation circuits for the simulated $P_{2}$. Choose $\widetilde{C} \leftarrow \mathbb{G}$ and send it to $P_{1}$. Receive in return $C \in \mathbb{G}$.

2. For each check circuit $j$, generate input-wire labels as in the real protocol. For each evaluation circuit $j$, choose $\widetilde{a}_{j, 1}, \ldots, \widetilde{a}_{j, n} \leftarrow \mathbb{Z}_{q}$ and set $\widetilde{v}_{j, i}=g^{\widetilde{a}_{j, i}}$ for $i=1, \ldots, n$. Also choose $\widetilde{v}_{j, n+i} \leftarrow\{0,1\}^{n}$ for $i=1, \ldots, n$.

When $P_{2}$ acts as OT receiver, run the OT protocol honestly using input 0. 
In each instance $i$ in which $P_{2}$ acts as OT sender, extract from $P_{1}$ (by rewinding the WI proof of knowledge) either $\log _{g} h_{i}$ or $\log _{g}\left(\widetilde{C} / h_{i}\right)$. In the former case set $x_{i}=0$ and in the latter case set $x_{i}=1$. Then, for check circuits send the final OT message exactly as in the real protocol, and for any evaluation circuit $j$ send the final OT message using $\widetilde{v}_{j, n+i}$ as the $x_{i^{-}}$ input, and the 0 -string as the $\left(1-x_{i}\right)$-input.

3 . Send $x$ to the trusted party, and receive in return an output $z$.

Generate $\left\{\widetilde{\operatorname{pub}}_{i}^{b}, \widetilde{w}_{j, i}^{b}\right\}$ as in the real protocol. Then for each evaluation circuit $j$, compute $\widetilde{\mathrm{GC}}_{j} \leftarrow \operatorname{SimGC}\left(x, z,\left\{\widetilde{v}_{j, i}\right\}_{i=1}^{2 n},\left\{\widetilde{w}_{j, i}^{z_{i}}\right\}_{i=1}^{n}\right)$; for each check circuit $j$, compute $\widetilde{\mathrm{GC}_{j}}$ as in the real protocol. Send $\left\{\widetilde{\mathrm{GC}}_{j}\right\}_{j=1}^{\kappa}$ and $\left\{\widetilde{\operatorname{pub}}_{i}^{0}, \widetilde{\operatorname{pub}}_{i}^{1}\right\}_{i=1}^{n}$ to $P_{1}$.

Receive in return $\left\{\mathrm{GC}_{j}\right\}_{j=1}^{\kappa}$ and $\left\{\operatorname{pub}_{i}^{0}, \operatorname{pub}_{i}^{1}\right\}_{i=1}^{n}$ from $P_{1}$.

4. For each check circuit $j$, compute $\left\{\widehat{\operatorname{ComSet}}_{j, i}\right\}_{i=1}^{n}$ as in the real protocol. For each evaluation circuit $j$, set $\widehat{\operatorname{ComSet}}_{j, i}=\left\{\operatorname{Com}\left(\widetilde{v}_{j, i}\right), \operatorname{Com}(g)\right\}$ in random permuted order. Send all these pairs of commitments to $P_{1}$, and receive in return all the pairs of commitments from $P_{1}$.

5. Give $P_{1}$ the value $\mathcal{J}$ as the output of the appropriate coin-tossing protocol. Respond for all check circuits as in the real protocol. For each evaluation circuit $j$, send $\left\{\widetilde{v}_{j, i}\right\}_{i=1}^{n}$, open the appropriate commitment from $\left\{\widehat{\operatorname{ComSet}}_{j, i}\right\}_{i=1}^{n}$, and send $\left\{\log _{g}\left(\widetilde{v}_{j, i} / \widetilde{h}_{i}\right)\right\}_{i=1}^{n}$, where $\widetilde{h}_{i}$ is the message sent by $P_{2}$ in the $i$ th OT when $P_{2}$ is receiver.

Choose $\widetilde{\mathcal{J}}$ at random as in the real protocol, and give it to $P_{1}$. If $P_{1}$ responds correctly, then repeatedly rewind to find $\widetilde{\mathcal{J}}^{\prime} \neq \widetilde{\mathcal{J}}$ for which $P_{1}$ responds correctly. (If none is found, $\mathcal{S}$ aborts with output fail.) Rewind again and continue the interaction using $\tilde{\mathcal{J}}$.

6. Let $j^{*}$ be a circuit which is an evaluation circuit in $\widetilde{\mathcal{J}}$, but a check circuit in $\widetilde{\mathcal{J}}^{\prime}$. For $i=1, \ldots, n$, use the $\kappa / 2$ shares of $s_{i}^{z_{i}}$ from $P_{1}$ 's check circuits (with respect to $\mathcal{J}$ ) plus the additional share of $s_{i}^{z_{i}}$ from circuit $j^{*}$ (that was a check circuit with respect to $\left.\widetilde{\mathcal{J}}^{\prime}\right)$ to reconstruct $s_{i}^{z_{i}}$. Set $\widetilde{t}_{i}^{z_{i}}=s_{i}^{z_{i}}$.

7. For $i=1, \ldots, n$, do the following.

- If $z_{i}=0$, obtain $P_{1}$ 's input $s_{i}^{0} \| t_{i}^{0}$ to the first equality test. If $s_{i}^{0} \| t_{i}^{0}=$ $\widetilde{t}_{i}^{0} \| \widetilde{s}_{i}^{0}$, return 1 ; else return 0 . Return 0 to the second equality test (if run).

- If $z_{i}=1$, return 0 to the first equality test. Then obtain $P_{1}$ 's input $s_{i}^{1} \| t_{i}^{1}$ to the second equality test. If $s_{i}^{1}\left\|t_{i}^{1}=\widetilde{t}_{i}^{1}\right\| \widetilde{s}_{i}^{1}$, return 1 ; else return 0 .

If for some $i$ both equality tests return 0 , abort. If an abort occurred, send abort to the trusted party; otherwise, send continue.

This completes the proof.

Acknowledgments. Yan Huang would like to thank Ivan Damgård and Jesper Nielsen for their helpful comments on this work. 


\section{References}

1. Aumann, Y., Lindell, Y.: Security against covert adversaries: Efficient protocols for realistic adversaries. Journal of Cryptology 23(2), 281-343 (2010)

2. Bellare, M., Garay, J.A., Rabin, T.: Fast batch verification for modular exponentiation and digital signatures. In: Nyberg, K. (ed.) EUROCRYPT 1998. LNCS, vol. 1403, pp. 236-250. Springer, Heidelberg (1998)

3. Canetti, R.: Security and composition of multiparty cryptographic protocols. Journal of Cryptology 13(1), 143-202 (2000)

4. Damgård, I., Keller, M., Larraia, E., Miles, C., Smart, N.P.: Implementing AES via an actively/Covertly secure dishonest-majority MPC protocol. In: Visconti, I., De Prisco, R. (eds.) SCN 2012. LNCS, vol. 7485, pp. 241-263. Springer, Heidelberg (2012)

5. Damgård, I., Pastro, V., Smart, N.P., Zakarias, S.: Multiparty computation from somewhat homomorphic encryption. In: Safavi-Naini, R. (ed.) CRYPTO 2012. LNCS, vol. 7417, pp. 643-662. Springer, Heidelberg (2012)

6. Feldman, P.: A practical scheme for non-interactive verifiable secret sharing. In: 28th Annual Symposium on Foundations of Computer Science (FOCS), pp. 427-437. IEEE (1987)

7. Goldreich, O.: Foundations of Cryptography. Basic Applications, vol. 2. Cambridge University Press, Cambridge (2004)

8. Goldreich, O., Micali, S., Wigderson, A.: How to play any mental game, or a completeness theorem for protocols with honest majority. In: 19th Annual ACM Symposium on Theory of Computing (STOC), pp. 218-229. ACM Press (1987)

9. Goyal, V., Mohassel, P., Smith, A.: Efficient two party and multi party computation against covert adversaries. In: Smart, N.P. (ed.) EUROCRYPT 2008. LNCS, vol. 4965, pp. 289-306. Springer, Heidelberg (2008)

10. Henecka, W., Kögl, S., Sadeghi, A.-R., Schneider, T., Wehrenberg, I.: TASTY: tool for automating secure two-party computations. In: 17th ACM Conf. on Computer and Communications Security (CCS), pp. 451-462. ACM Press (2010)

11. Huang, Y., Evans, D., Katz, J.: Private set intersection: Are garbled circuits better than custom protocols? In: Network and Distributed System Security Symposium (NDSS). The Internet Society (2012)

12. Huang, Y., Evans, D., Katz, J., Malka, L.: Faster secure two-party computation using garbled circuits. In: 20th USENIX Security Symposium. USENIX Association (2011)

13. Huang, Y., Katz, J., Evans, D.: Quid pro quo-tocols: Strengthening semi-honest protocols with dual execution. In: IEEE Symposium on Security \& Privacy, pp. 239-254. IEEE (2012)

14. Ishai, Y., Prabhakaran, M., Sahai, A.: Founding cryptography on oblivious transfer - efficiently. In: Wagner, D. (ed.) CRYPTO 2008. LNCS, vol. 5157, pp. 572-591. Springer, Heidelberg (2008)

15. Jarecki, S., Shmatikov, V.: Efficient two-party secure computation on committed inputs. In: Naor, M. (ed.) EUROCRYPT 2007. LNCS, vol. 4515, pp. 97-114. Springer, Heidelberg (2007)

16. Kiraz, M., Schoenmakers, B.: A protocol issue for the malicious case of yao's garbled-circuit construction. In: Proc. 27th Symp. on Information Theory in the Benelux, pp. 283-290 (2006) 
17. Kolesnikov, V., Schneider, T.: Improved garbled circuit: Free XOR gates and applications. In: Aceto, L., Damgård, I., Goldberg, L.A., Halldórsson, M.M., Ingólfsdóttir, A., Walukiewicz, I. (eds.) ICALP 2008, Part II. LNCS, vol. 5126, pp. 486-498. Springer, Heidelberg (2008)

18. Kreuter, B., Shelat, A., Shen, C.: Billion-gate secure computation with malicious adversaries. In: 21st USENIX Security Symposium. USENIX Association (2012)

19. Lindell, Y.: Fast cut-and-choose based protocols for malicious and covert adversaries. In: Canetti, R., Garay, J.A. (eds.) CRYPTO 2013, Part II. LNCS, vol. 8043, pp. 1-17. Springer, Heidelberg (2013)

20. Lindell, Y., Oxman, E., Pinkas, B.: The IPS compiler: Optimizations, variants and concrete efficiency. In: Rogaway, P. (ed.) CRYPTO 2011. LNCS, vol. 6841, pp. 259-276. Springer, Heidelberg (2011)

21. Lindell, Y., Pinkas, B.: An efficient protocol for secure two-party computation in the presence of malicious adversaries. In: Naor, M. (ed.) EUROCRYPT 2007. LNCS, vol. 4515, pp. 52-78. Springer, Heidelberg (2007)

22. Lindell, Y., Pinkas, B.: A proof of security of Yao's protocol for two-party computation. Journal of Cryptology 22(2), 161-188 (2009)

23. Lindell, Y., Pinkas, B.: Secure two-party computation via cut-and-choose oblivious transfer. Journal of Cryptology 25(4), 680-722 (2012)

24. Lindell, Y., Pinkas, B., Smart, N.P.: Implementing two-party computation efficiently with security against malicious adversaries. In: Ostrovsky, R., De Prisco, R., Visconti, I. (eds.) SCN 2008. LNCS, vol. 5229, pp. 2-20. Springer, Heidelberg (2008)

25. Malkhi, D., Nisan, N., Pinkas, B., Sella, Y.: Fairplay - a secure two-party computation system. In: Proc. 13th USENIX Security Symposium, pp. 287-302. USENIX Association (2004)

26. Mohassel, P., Franklin, M.: Efficiency tradeoffs for malicious two-party computation. In: Yung, M., Dodis, Y., Kiayias, A., Malkin, T. (eds.) PKC 2006. LNCS, vol. 3958, pp. 458-473. Springer, Heidelberg (2006)

27. Naor, M., Pinkas, B.: Efficient oblivious transfer protocols. In: 12th SODA Annual ACM-SIAM Symposium on Discrete Algorithms (SODA), pp. 448-457. ACMSIAM (2001)

28. Naor, M., Pinkas, B., Sumner, R.: Privacy preserving auctions and mechanism design. In: Proc. 1st ACM Conf. on Electronic Commerce, pp. 129-139. ACM (1999)

29. Nielsen, J.B., Nordholt, P.S., Orlandi, C., Burra, S.S.: A new approach to practical active-secure two-party computation. In: Safavi-Naini, R. (ed.) CRYPTO 2012. LNCS, vol. 7417, pp. 681-700. Springer, Heidelberg (2012)

30. Nielsen, J.B., Orlandi, C.: LEGO for two-party secure computation. In: Reingold, O. (ed.) TCC 2009. LNCS, vol. 5444, pp. 368-386. Springer, Heidelberg (2009)

31. Peikert, C., Vaikuntanathan, V., Waters, B.: A framework for efficient and composable oblivious transfer. In: Wagner, D. (ed.) CRYPTO 2008. LNCS, vol. 5157, pp. 554-571. Springer, Heidelberg (2008)

32. Pinkas, B., Schneider, T., Smart, N., Williams, S.: Secure two-party computation is practical. In: Matsui, M. (ed.) ASIACRYPT 2009. LNCS, vol. 5912, pp. 250-267. Springer, Heidelberg (2009)

33. Shelat, A., Shen, C.-H.: Two-output secure computation with malicious adversaries. In: Paterson, K.G. (ed.) EUROCRYPT 2011. LNCS, vol. 6632, pp. 386-405. Springer, Heidelberg (2011) 
34. Woodruff, D.P.: Revisiting the efficiency of malicious two-party computation. In: Naor, M. (ed.) EUROCRYPT 2007. LNCS, vol. 4515, pp. 79-96. Springer, Heidelberg (2007)

35. Yao, A.C.-C.: How to generate and exchange secrets. In: 27th Annual Symposium on Foundations of Computer Science (FOCS), pp. 162-167. IEEE (1986)

\section{A Experimental Results}

We describe some preliminary experimentals indicating that our protocol significantly outperforms the recent work of [18].

We implemented our protocol in Java using all the optimizations of Section 4.1. We evaluated the protocol at the 80-bit security level, which means in particular that (1) each party generates 84 garbled circuits, 42 of which are checked; (2) the length of all wire labels is 80 bits; and (3) we use an order- $q$ subgroup of $\mathbb{Z}_{p}^{*}$ where $|p|=1024,|q|=160$. We ran experiments over a LAN using two laptops with Intel Core i7 $2.4 \mathrm{GHz}$ processors. Note that 80 -bit security was also used in the experiments of [18.

In typical settings where the number of gates in the underlying circuit is much larger than the number of inputs/outputs, the dominant overall cost of the protocol is the generation, sending, and checking of the garbled circuits. When each side uses only a single core, our protocol evaluates circuits at the rate of $1.4 \mathrm{~ms}$ /gate. By comparison, the implementation of Kreuter et al. [18] evaluates circuits at the rate of about $8 \mathrm{~ms} /$ gate when a single thread is used.

When each side utilizes two cores, our protocol evaluates circuits at the rate of $0.8 \mathrm{~ms} /$ gate; by comparison, the two-threaded execution in [18] achieved a rate of roughly $4 \mathrm{~ms} /$ gate. We do not gain a factor of 2 in performance by leveraging a second core in part because the parties are sometimes idle, and in part because of inter-thread interference (e.g., due to cache contention and dependence on shared hardware and $\mathrm{I} / \mathrm{O})$.

Our measured performance gains relative to [18] exceed the expected factor of 3. This may be due to differences in hardware or implementation, or the complexity of managing multiple threads in the implementation of [18] regardless of how many cores are being used.

The number of public-key operations used in our protocol scales linearly with the lengths of the inputs and outputs, though we stress again that in typical scenarios the number of gates is much larger than the number of inputs/outputs and so the overall performance impact of these public-key operations is small. Nevertheless, we measured performance of this aspect of our protocol as well. When each side uses a single core, our protocol processes inputs at the rate of $0.7 \mathrm{~s} /$ bit (our experiments assume the lengths of the parties' inputs are the same). Output is computed at the rate of $0.1 \mathrm{~s} / \mathrm{bit}$. 